\title{
Introduction to OSS 2007 Tutorial Program
}

\author{
Ernesto Damiani \\ DTI - University of Milan, Italy \\ damiani@dti.unimi.it
}

Today, most software researchers and professionals consider Open Source as a greatly successful paradigm for large-scale production of high-quality software systems. However, most of them would probably agree that successfully applying this paradigm requires dealing with a number of important issues. One of the best reasons to attend OSS conference series is being able to discuss these issues in an unprecedented gathering of top-notch researchers, developers, and leaders from all avenues of the open source movement. Attending good tutorials, in particular, is a great opportunity for getting in touch with new ideas and discussing them in depth with real experts.

For this reason, I am especially glad to introduce OSS 2007 tutorial program, which in my opinion does worthily complete the conference's rich program of research sessions and workshops. The aim of OSS tutorial series is to simply explain complicated subjects, attracting an audience whose composition will hopefully mirror the one of most free and open source software communities today, including absolute beginners as well as experienced open source gurus.

This year's rigorous selection process has selected an extraordinary set of tutorials which provide straightforward, but never trivial, introductions to a wide range of topics concerning the emerging role of open source in a number of application domains.

Namely, Megan Conklin's tutorial "How to Gather Metrics on FLOSS projects", will introduce the crucial problem of representing and gathering knowledge on FLOSS as a prerequisite to advanced decision making, e.g. on open source software adoption issues.

François Déchelle's tutorial "EDOS Tools for Linux Distributions Dependencies Management and Quality Assurance" will discuss a number of relevant issues related to testing and quality assurance of heterogeneous open source packages and present tools developed in the EDOS project for managing Linux distribution testing and quality assurance processes.

Finally, Hans-Ludwig Hausen's tutorial "Quality Specification, Testing and Certification of Bespoken, Open Source and Commercial Off-The-Shelf Systems" addresses some important software quality and metrology methods and procedures (specification, testing, V\&V, reliability, safety, security, measurement, assessment,

Please use the following format when citing this chapter:

Damiani, E., 2007, in IFIP International Federation for Information Processing, Volume 234, Open Source Development, Adoption and Innovation, eds. J. Feller, Fitzgerald, B., Scacchi, W., Sillitti, A., (Boston: Springer), pp. 359-360. 
etc) suitable for dependable information system engineering and acceptance testing or for software certification.

The tutorial section in OSS 2007 Proceedings includes concise summaries of these three accepted tutorials, hopefully conveying some of the spirit of the lively exchange of ideas these tutorials will encourage at OSS 2007.

Putting together an attractive and scientifically sound tutorial program like this one is always a team effort. I would like to thank all OSS 2007 officials for their valuable comments and help in the selection process. Special thanks are also due to Alberto Colombo and Fulvio Frati, OSS 2007 Web masters, for their help in making the selection process as smooth and efficient as one might desire. Above all, I wish to thank all the tutorial proposers for choosing OSS 2007 as the preferred venue for presenting their ideas and techniques, and the tutorial attendees whose participation and feedback are essential for making OSS 2007 tutorial program a success. 